\title{
p97 /VCP and Inclusion Body Myopathy with Early-Onset Paget Disease and Frontotemporal Dementia (IBMPFD)
}

\author{
Yalçın Erzurumlu (D) \\ Department of Biochemistry, Faculty of Pharmacy, Süleyman Demirel University, Isparta, Turkey
}

ORCID iD of the author: Y. E. 0000-000I-6835-4436.

Cite this article as: Erzurumlu Y. p97 /VCP and Inclusion Body Myopathy with Early-Onset Paget Disease and Frontotemporal Dementia (IBMPFD). Cyprus J Med Sci. 2021; 6(4): 337-344.

\begin{abstract}
Inclusion body myopathy with early-onset Paget disease and frontotemporal dementia (IBMPFD), which is an autosomal dominant inheritance multisystem disease, is rare, occurring in adulthood, progressive progression, and often results in the death of patients. Clinically, IBMPFD is characterized by limb-girdle muscle dystrophy syndrome sighted proximal and distal muscle weakness, early-onset bone Paget's disease, and premature frontotemporal dementia. First, in 2004, the IBMPFD disease locus was mapped at the p2I-p12 locus of chromosome 9 and was associated with mutations observed in the p97 /VCP gene. Biologically, p97/VCP has been shown to have a regulatory and catalyzing role in many cellular processes, including postmitotic homotypic membrane fusion, nuclear sheath regeneration and packaging, cell cycle regulation, programed cell death, endoplasmic reticulum-associated degradation, organelle biogenesis, regulation of transcription factors, Endoplasmic reticulum membrane fusion, stimulation of B and T cells, and inhibition and separation of protein aggregates. IBMPFD has been associated with mutations observed in the p97/VCP gene. Currently, the number of p97 NCP mutations identified in IBMPFD patients is 28. The molecular mechanisms of only a few of these mutations in IBMPFD pathogenesis were understood. In this review, all of the p97 /VCP mutations associated with IBMPFD are discussed, and the molecular mechanisms of p97/VCP in disease process are examined.
\end{abstract}

Keywords: p97 NCP, inclusion body myopathy with early-onset Paget disease and frontotemporal dementia, endoplasmic reticulumassociated degradation

\section{INTRODUCTION \\ Inclusion Body Myopathy with Early-Onset Paget Disease and Frontotemporal Dementia}

Inclusion body myopathy with early-onset Paget disease and frontotemporal dementia (IBMPFD; OMIM \#605382) is rare, occurring in adulthood, gradually progression, and often results in the death of patients. IBMPFD has autosomal dominant inheritance and onset in adults between 20 and 40 years of age. IBMPFD was mapped at 9p21.I-pl2 locus of chromosome 9. This locus, which is very rich in gene diversity, is characterized as a multiple disease locus with different myopathies. IBMPFD is observed with myopathy, Paget's disease, and dementia in various penetrations. ${ }^{1,2}$

Clinically, IBMPFD is characterized by limb-girdle muscle dystrophy syndrome sighted proximal and distal muscle weakness, early-onset bone Paget's disease (PDB), and premature frontotemporal dementia (FTD). The first symptoms of the disease are observed in the brain and bone tissue. ${ }^{3}$ The muscle weakness observed in the patients gradually progressed, affecting the muscles of the other limbs and respiratory tract. It has also been reported that deformation and enlargement of long bones and occasionally pathological fractures of the bones could be seen in some patients. Currently, dilated cardiomyopathy, amyotrophic lateral sclerosis (ALS), and Parkinson's disease are known to be part of the spectrum of findings associated with IBMPFD. ${ }^{4}$

IBMPFD was first reported in 2004 due to mutations in the gene encoding valosin-containing protein (VCP and p97) VCP) located at pl3-pl2 locus of chromosome 9.,2 p97/VCP is known as a molecular chaperone that plays an active role in many cellular processes. ${ }^{5}$ The first symptoms of IBMPFD are observed in brain and bone tissue. ${ }^{3}$ Typically, clinical muscle phenotype seen in IBMPFD patients is characterized by weakness in the lower extremities and upper proximal, often with axial myopathy, wing-like extensions of the shoulder blades, and variable involvement of the distal muscles. It 
has been observed that respiratory failure and cardiomyopathy findings are encountered in some patients due to myopathy.

Inclusion body myopathy (IBM) with autosomal dominant inheritance exhibits a heterogeneous course profile, in which clinical reflections and genetic disorders are observed. IBM is characterized by late-onset shoulder and pelvic weakness in patients and creatine kinase (CK) levels that are clinically above the desired range. The reflection of myopathic changes in patients' cells is observed as cytoplasm, and more rarely, vacuoles observed in the nucleus of cells. ${ }^{6}$

PDB is more common in male individuals than in women. The pathology of the disease is characterized by increased resorption of bone by osteoclasts and subsequent reshaping of bone formation. Although it is generally asymptomatic, it is characterized by abnormal, overactive osteoclasts, bone thickening, and usually large, excessively large nuclei and often paramyxovirus-like inclusions. Ubiquitinated cytoplasmic and nuclear inclusions were observed in pagetoid osteoclasts. PDB was found to occur at similar ages with myopathy in $50 \%$ of IBMPFD patients. ${ }^{3}$ In PDB patients, clinical symptoms are observed, including bone deformation, spine, hip and skull pain, and bone fractures. Increased alkaline phosphatase enzyme levels were also determined in patients. ${ }^{2}$

FTD in IBMPFD patients is an important part of primary degenerative dementia before the age of 65. FTD is observed as frontal lobe functions associated with disproportionately weakened behavioral changes. It is observed that memory, visual, and spatial abilities are partially preserved in the early stages of the disease, but the symptoms strongly support the diagnosis of FTD. The underlying cause of all these symptoms is due to the changes observed in the frontal lobe. Patients also have localized atrophy of the frontal and anterior temporal lobes. 38-45\% of all FTD cases have a strong hereditary transmission, and approximately $80 \%$ of this group has an autosomal dominant inheritance. ${ }^{6}$ In IBMPFD patients, the penetrance of FTD was determined to be $30 \%$, and it was determined that the onset of FTD occurred much later than myopathy. ${ }^{3}$

Further studies show that cytosolic and intranuclear sections of IBMPFD tissue were found to be significantly ubiquitinated inclusions. $^{7-9}$ In the studies that healthy muscle samples were used as a control group, it was observed that p97 / VCP was localized in the endomysial capillary structure, which is mostly the structure of the ligament layer in muscle by staining with polyclonal primer antibody. The formation of ubiquitinated small inclusions and vacuolar structures in the cytosolic segments of muscle fibers of IBM patients was determined. In the same examples, p97 / VCP was found to be strongly involved in endomysial inflammatory cells surrounding muscle fibers. Besides, p97 / VCP levels increased in the regenerated muscle fibers, and p97 /VCP was found to be involved in wide focal inclusions of muscle fibers belonging to IBMPFD patients. ${ }^{10}$

When IBMPFD was examined in terms of central nervous system pathology, it was observed that these cells were negative for tau protein and positive for ubiquitin, consistent with ubiquitinated inclusions and frontotemporal lobar degeneration." IBMPFD is characterized by disturbances in the degradation of the accumulation of proteins, and ubiquitinated protein aggregates targeted by p97/VCP., ${ }^{9-12}$ Cellular degeneration and ubiquitinated protein inclusions combine the pathology of these three separate tissues (bone, muscle, and brain) in IBMPFD. It is known that p97 / VCP is a multifunctional protein and plays a major role as a molecular chaperone in the transfer of proteins that unfolded and misfolded proteins in the ER. ${ }^{10}$ Furthermore, the pathogenicity exhibited by p97/VCP is emphasized by p97/VCP positive protein aggregates observed in neurons and skeletal muscle cells located in the central nervous system of IBMPFD patients. ${ }^{7}$ It has been reported that $\mathrm{p} 97$ / $\mathrm{CP}$ positive inclusions are mainly concentrated in the nucleus of neuron cells in IBMPFD patients, and only in cytoplasmic aggregate foci in skeletal muscle cells. ${ }^{9}$

Nuclear inclusions containing ubiquitin were found to be colocalized with p97 NCP in various neurodegenerative disorders such as Huntington's disease, Lewy body disease, Parkinson's disease, spinocerebellar ataxia type III (Machado-Joseph disease), and ALS. ${ }^{1,9}$ In studies conducted by different groups, these aggregates, which were positive for $\mathrm{p} 97$ / VCP, were not reported to be specific to IBMPFD pathology. Similarly, different proteins such as ubiquitin, $\beta$-amyloid, apolipoprotein $\mathrm{E}$, and phospho-Tau in the cytoplasmic aggregates in the muscles of IBMPFD patients were found to accumulate in the brain cells of Alzheimer's patients. ${ }^{12}$

The phenotypically related features of IBMPFD are also found in $90 \%$ of patients and are often characterized in the $40 \mathrm{~s}$, which is the adulthood of individuals. Atrophy associated with proximal and distal muscle weakness is observed in these individuals. In skeletal muscles with IBMPFD pathology, amorphous vacuoles (rimmed vacuoles), myonuclear, and sarcoplasmic inclusions have been reported. These inclusions have been reported to be congophilic and immunoreactive for TAR DNA binding protein-43 (TDP-43) in some cases. ${ }^{3}$

In the molecular pathogenesis of IBMPFD, p97/VCP is critical due to the role in targeting the proteasome of unfolded and misfolded proteins that may cause proteotoxicity by means of endoplasmic reticulum (ER)-associated degradation (ERAD). Therefore, it is of great importance to understand the biological role of p97 / VCP and associating it with the pathogenesis of IBMPFD.

\section{Valosin Containing Protein (p97/VCP)}

p97/VCP is encoded in the pl3.3 locus of chromosome 9 and localized in the negative (-) strand of the genome. The 17 exons p97 / VCP are encoded by a 3859 bp transcript. Consisting of 806 amino acids, p97/VCP has a molecular weight of about $89,322 \mathrm{Da}$. The calculated basal isoelectric point is known to be $5.14 .^{15}$

The p97/VCP protein was first identified as the protein responsible for cell division control in yeast under the name CDC48, while it was reported as provalosin in metazoans. The sequence analysis revealed that the yeast and human VCP gene had a similarity of $69 \%$. In the evolutionary process, p97/ VCP was found to be highly conserved between diverse species. In sequence homology studies, it was observed that p97/ VCP showed advanced homology among different species. p97/VCP, VAT in Archaebacteria (VCP-like ATPase), CDC48p in Saccharomyces cerevisiae (cell division control protein 48), TER94 (transitional endoplasmic reticulum ATPase) in 


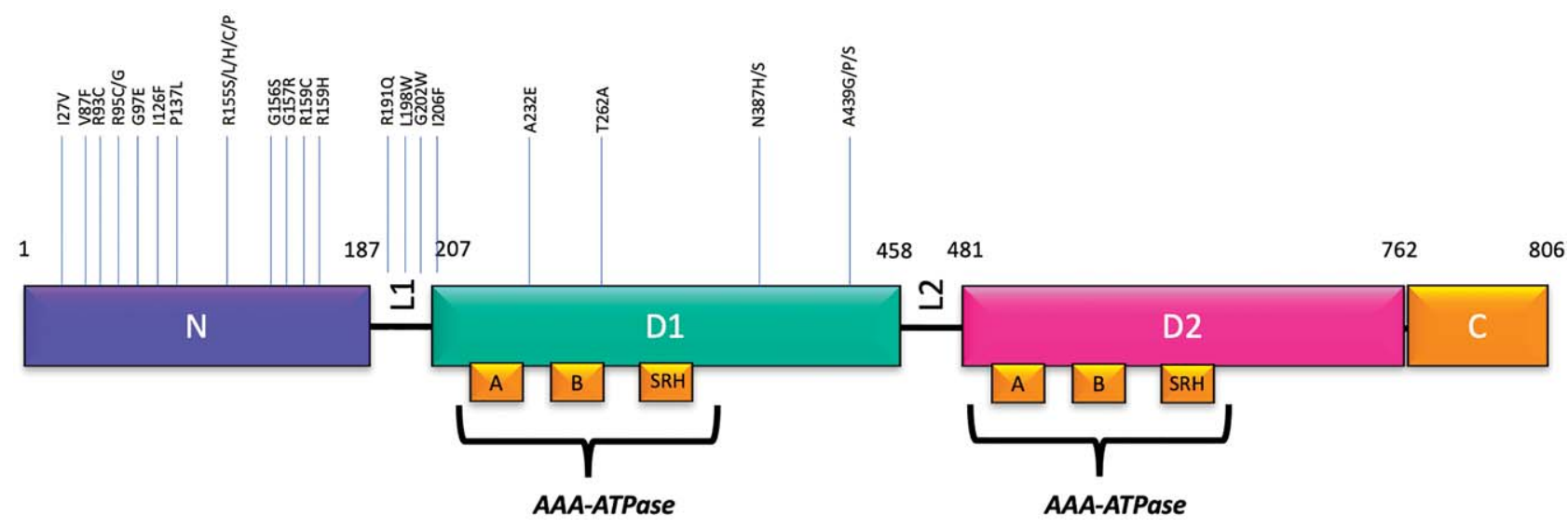

Figure I. Protein organization of p97 /VCP and localization of IBMPFD-related p97/VCP mutations. Schematic organization of a p97/VCP protein structure showing the three domains: N-terminal N domain and two ATPase domains in DI and D2, and the positions of IBMPFD-related mutations.

Drosophila melanogaster, p97 in Xenopus laevis, and plant and mammalian species orthologs are named as VCP. In Northern blot analyses, it was determined that p97/VCP was expressed in all tissues in the body, including brain tissue in humans. ${ }^{16}$ Also, p97 / VCP, which constitutes more than $1 \%$ of the protein content in the cells, was found to be ubiquitously expressed in cells and be in large amounts of cytosolic compartments. ${ }^{17}$ These data highlight the critical role of p97/VCP in living systems.

Biologically, p97/VCP has been shown to have a regulatory and catalyzing role in many cellular processes, which include postmitotic homotypic membrane fusion, nuclear sheath regeneration, packaging, cell cycle regulation, programed cell death, ERAD, organelle biogenesis, mitotic spindle separation, regulation of transcription factors, ER membrane fusion, stimulation of $B$ and $T$ cells, inhibition and separation of protein aggregates, DNA repair, and autophagy. ${ }^{18}$

Each unit of p97/VCP, which consists of six identic subunits with each other and exhibits a homo-hexameric structure, has four different domains known to be important for maintaining its functionality. These are N-domain (I-187), DI domain (209460), D2 domain (48I-76I), C-terminal domain (762-806), N-DI linker LI region (I88-208), and flexible DI-D2 linker L2 region (461-480). ${ }^{19,20}$ Electron micrography studies have shown that p97 / VCP exhibits two homo-hexameric structures organized as barrels. Also, it has been indicated in crystallographic studies that the p97/VCP hexamer exhibits a flattened hourglass or mushroom-like structure. ${ }^{20}$

A good understanding of protein organization is essential for comprehending the role of p97 / VCP in biological systems. The amino (N) and carboxyl (C) terminal domains of p97/VCP mediate interaction with various adapter and accessory proteins. It has been shown that the N-terminal domain is critical for interaction with several cofactors for cellular position and activity and also for substrate interactions. ${ }^{21}$ The C-terminal domain of p97 NCP has flexible acidic amino acid residues. Defects in the C-terminal domain have been shown to cause changes in cellular responses due to the change in binding of cofactors that provide substrate modifications. Furthermore, the C-terminal domain contains a major tyrosine phosphoryla- tion residue (Tyr805) having a regulatory role. ${ }^{22}$ Phosphorylation in this region regulates protein interactions by inhibiting cofactor binding to p97/ VCP. ${ }^{23}$ Also, the ATPase cassettes required for the biological activity of $\mathrm{P} 97 / \mathrm{VCP}$ are located in the domains DI and D2, respectively. ${ }^{22}$ p 97 / VCP is a member of the ATPase superfamily of type II AAA + (ATPases-associated with diverse Activities) involved in multiple cellular pathways. AAA + proteins are categorized into two classes based on the number of conserved ATP-binding cassettes contained in the protein structure. While class I AAA + ATPase family member proteins have one AAA + cassette in their structure, class II members have two AAA + cassettes. p97/VCP, a member of the Class II superfamily, performs the mechanical role in biological events by the energy obtained from the hydrolysis of ATP using ATPase cassettes. ${ }^{23}$ In homology studies, each AAA + domain of p97/VCP has a high sequence similarity among different species. This supports that AAA + proteins, including P97 / VCP, are extremely important structural elements in biological systems for survival. ${ }^{24}$

The AAA+ domains of ATP binding cassettes are well protected in the evolutionary process. AAA + cassettes consist of Walker A and Walker B motif, sensors I and 2, the second region of homology $(\mathrm{SRH})$, and the pore loop. Walker $A$ motifs having the conserved GxxxxGK(T/S) sequence are necessary for nucleotide binding. Walker $B$ motifs having ( $R$ / $\mathrm{K})$ xxxGxxx(L/V)hhh(D/E) have been determined to have the conserved sequence. It has been reported that the ATPase cassette of the Walker $\mathrm{B}$ and $\mathrm{SRH}$ regions are necessary for efficient ATP hydrolysis. It has been reported that mutations in Sensor I cause defects in hydrolysis function. In addition, a conserved arginine residue of Sensor 2 directly interacts with the g-phosphate group of ATP via the neighboring subunit, and this interaction is necessary for ATP binding and hydrolysis. It has been indicated that the $\mathrm{SRH}$ region contains the critical arginine fingers, which is important in interaction, and the pore loop region is required for binding to and processing of the substrate (Figure I). ${ }^{25}$

Allosteric interactions are very essential for the function of p97 / VCP, which has a multimeric structure. ATP binding to the ATPase cassette in the DI domain of p97 / VCP was found to accelerate the reassociation of $\mathrm{p} 97 / \mathrm{VCP}$ monomers. This 


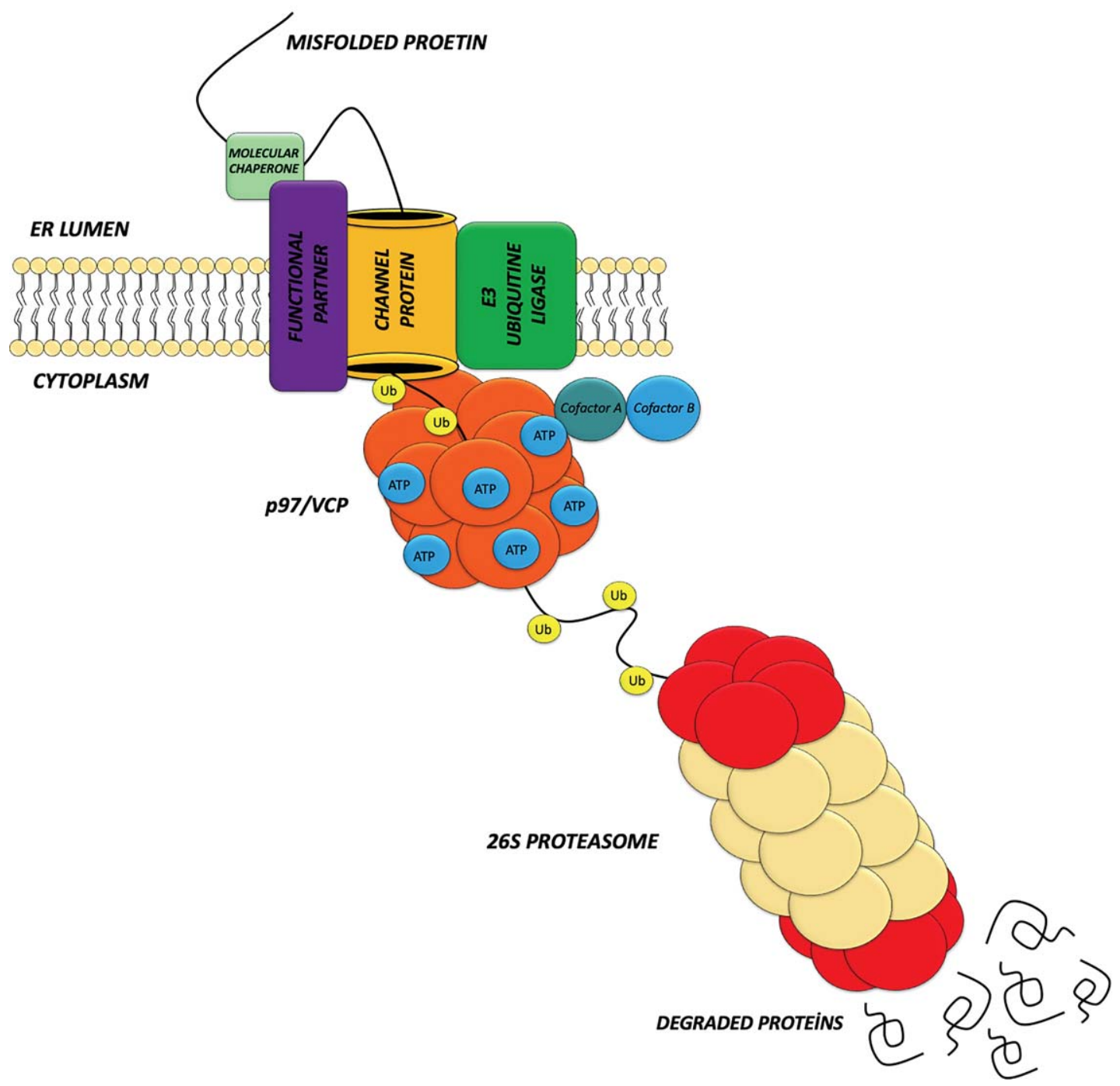

Figure 2. A model for the direction of misfolded proteins in ER to $26 \mathrm{~S}$ proteasome. Primarily, misfolded proteins are recognized by luminal molecular chaperone complex and targeted to a putative channel in the membrane. The partially unfolded substrate is dislocated across the ER lumen simultaneously; ubiquitylation occurs through membrane-bound substrate-specific E3 ubiquitin ligases, facilitating recruitment of p97/VCP. p97 $/ \mathrm{VCP}$, with its ATPase activity, provides the transfer of misfolded protein from the channel protein to the cytosolic face of ER. The misfolded protein is then directed to different protein complexes the $26 \mathrm{~S}$ proteasome and degraded.

result suggests that DI domain is an important tool for hexamerization. DI domain has a major role in oligomerization of protein, whereas D2 domain has a minor effect in this process. The formation of the hexameric structure is very important for the biological functions of $\mathrm{p} 97$ / VCP, especially critical for its role in the ubiquitin-proteasome system. ${ }^{20}$ The mutations seen in Walkers A and B motifs in D2 domain of p97 / VCP were predominantly fatal in individuals, and mutations in DI domain did not have such an effect. ${ }^{24}$ Functional distortions in $\mathrm{p} 97$ / VCP trigger dysregulated processes, such as impaired protein quality control, mitochondrial dysfunction, oxidative stress, and apoptotic cell death resulting in cell death. ${ }^{26}$

p97/VCP is a key molecule involved in the retrotranslocation step of ERAD. ${ }^{27}$ Unfolded, incorrectly oligomerized and misfolded proteins are potentially proteotoxic to the cells. Newly synthesized proteins and synthesized proteins cannot reach their mature forms due to various reasons, such as genetic mutation, viral infection, temperature changes, and oxidative stress. Evolutionary, mammalian cells have developed qualitycontrol systems for the removal of these abnormal proteins. ${ }^{28}$ These systems only allow proteins that reach their final conformational state to have them move to their sites. Newly synthesized proteins in the ER are continuously monitored by the various quality control system. Unwanted abnormal protein forms are targeted to $26 \mathrm{~S}$ proteasome by ERAD mechanism. ${ }^{28}$ ERAD also functions to regulate endogenous levels of diverse proteins, including cholesterol synthesis-associated enzyme 3Hydroxy-3-Methylglutaryl-CoA Reductase and tumor suppressor KAll. Thus, ERAD may regulate the cellular homeostasis and adapt the cells to changing physiological conditions.

The ubiquitin molecule is conjugated to the misfolded proteins in the ER by ubiquitin ligase enzymes. p97 / VCP interacts with ubiquitinated proteins on the cytosolic surface of the ER lumen, performing retrotranslocation of these proteins from ER to cytosol (Figure 2). ${ }^{27}$ Mutations observed in p97 / VCP and the factors impairing its functionality prevent the removal of these 


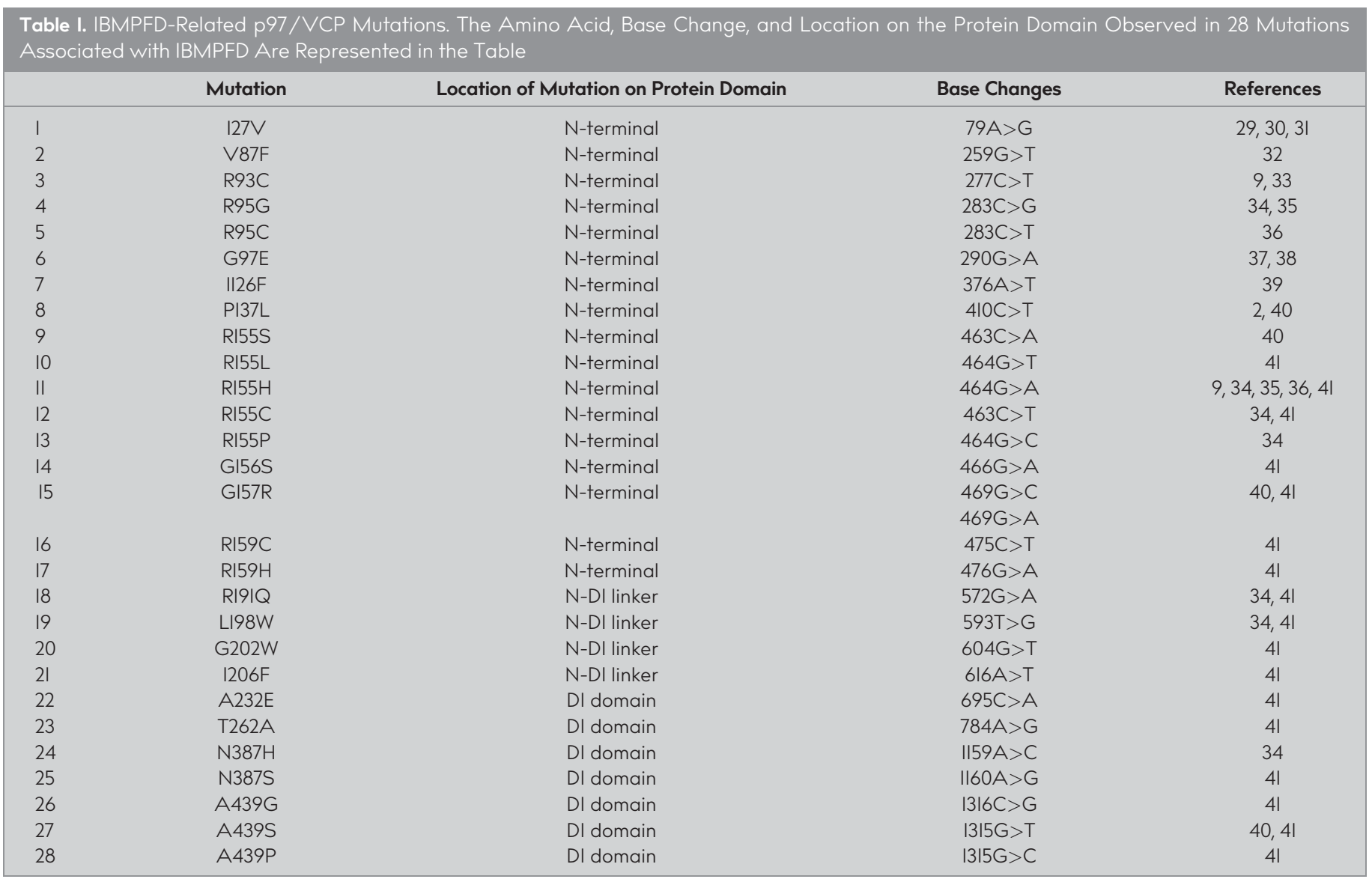

unwanted proteins from the cells. Given that the protein aggregates accumulated in cells are caused by the molecular pathogenesis of IBMPFD, functional losses in p97 / VCP will result in the accumulation of ubiquitinated proteins of cells, the formation of protein aggregates, and also the induction of ER stress. When these protein aggregates that cause cellular damage cannot be overcome, programmed cell death is triggered by increased cellular stress. ${ }^{29}$ It is known that p97 / VCP is associated with many functional disorders. Especially, increasing our knowledge about p97/VCP biology is important for understanding the role of pathogenesis in IBMPFD.

\section{Functional Role of p97 /VCP in IBMPFD Pathogenesis: Molecular view}

The IBMPFD disease locus with multiple system disease was mapped at the p2l-pl2 locus of chromosome 9 and was associated with mutations observed in the p97 / VCP gene. To date, the number of p97/VCP mutations associated with the pathogenesis of IBMPFD in patients has been identified as 28 (Table I). ${ }^{2,29-4 I}$ However, the details of only a few of these mutations have been revealed in the molecular pathogenesis of IBMPFD.

The point mutations associated with IBMPFD were found in three different domains of p97 / VCP.

The mutations that are 127V, V87F, R93C, R95C/G, G97E, II26F, PI37L, RI55H/P/C/S/L, GI56S, GI57R, and RI59H/C in the Nterminal domain, RI9IQ, LI98W, G202W, and I206F in the N-DI link domain, and A232E, T262A, N387H/S, and A439G/P/S in the DI ATPase domain were identified in IBMPFD patients. To date, 28 missense mutations associated with IBMPFD have been demonstrated in $\mathrm{P} 97 / \mathrm{VCP}{ }^{2,29-41}$ The most common point mutation in IBMPFD patients is the amino acid of arginine at position 155.,30,42 Fernández-Sáiz and Buchberger" found that IBMPFD-related R95G and RI55H mutations lead to conformational changes in the N-terminal domain of p97 / VCP, and thus weakened the interaction between the $\mathrm{N}$ and $\mathrm{DI}$ domains. It has been reported that these mutations lead to changes in the interaction of $\mathrm{p} 97$ / VCP with cofactors and are important in the pathology of IBMPFD. In 2007, Issacson et al. identified the interaction pattern between p97/VCP and Npl4, thereby using nuclear magnetic resonance spectroscopy methodology. Some point mutations associated with IBMPFD (R93, R95, and RI55) have been reported to be located in the region where p97 / VCP interacts with Npl4. As a result of these point mutations, it is shown that functional losses may occur in the ERAD pathway because of the difference or deterioration in the interaction pattern of the Ufdl-Npl4 cofactor complex and p97/VCP. ${ }^{43}$ In the studies conducted with R95G mutation in patients, a double $\psi$ barrel structure of p97 $\mathrm{VCP}$ was found to be destructured. ${ }^{34}$ In cell culture models, it was determined that R95G mutant caused a significant increase in ubiquitin conjugated protein levels. Functionally, these results suggest that the R95G mutant p97 / VCP weakens proteasomal degradation of substrates molecules. ${ }^{2}$ Furthermore, ubiquitinated proteins and transgenic IBMPFD mouse models were determined to accumulate in cell culture after the suppression of p97 / VCP with RNA interference-mediated silencing or pharmacologically inhibition. Similar results have been obtained in 
IBMPFD-related R95G, RI55H, L198W, and A232E p97/VCP mutant protein expressing cells. ${ }^{3}$ These results suggest that p97 / VCP mutants lead to the disruption of functional ERAD in IBMPFD patients. In the protein structure studies, it was reported that mutations of RI55C, RI55H, and RI55P located at the N-terminus of p97 / VCP disrupt the folding of four strands $\beta$ barrel protein of $\mathrm{p} 97 / \mathrm{VCP}$. In the studies conducted with RI9IQ mutant protein, deterioration has been found in the organization of the flexible binding region. ${ }^{10}$ The LI98W mutation has been determined stoichiometrically to disrupt the normal movement of the N-terminal of p97/VCP. This mutation causes conformational change at the N-terminal of p97/ VCP and leads to changes in protein interactions via this domain. ${ }^{42}$ The A232E mutation has been reported to affect the $\alpha 5$-helix structure in the $a / b$ subdomains of DI involved in the ATPase domain of p97 / VCP. This ATPase domain is responsible for the catalytic activity of p97/VCP and the formation of the hexameric protein form. Therefore, it suggests that the destructive effect of $\mathrm{A} 232 \mathrm{E}$ mutation in the pathogenesis of IBMPFD has been stronger. ${ }^{34}$

In the myoblast cell culture $(\mathrm{C} 2 \mathrm{Cl} 2)$ model, after the ectopic expression of $\mathrm{RI} 55 \mathrm{H}$ or R95G mutant proteins, steady-state levels of mutant cystic fibrosis transmembrane conductance regulator CFTR $\Delta$ F508 protein, which is degraded by targeting to the proteasome by ERAD, increased. ${ }^{3}$ However, $\mathrm{RI} 55 \mathrm{H}$ and A232E p97/ VCP mutant proteins did not affect the proteasomal degradation of another well-known ERAD substrate CD3 $\delta .^{12}$ These results suggest that all IBMPFD-associated p97/ VCP mutations exhibit specific characteristic patterns in the ERAD or ubiquitin-proteasome system. Therefore, it is important to determine the mechanistic details of all the p97/ VCP mutations associated with IBMPFD at the level of protein interactions.

Erzurumlu et al. ${ }^{44}$ examined the effect of 12 IBMPFD-associated p97 NCP mutations, including R93C, R95G, PI37L, RI55C, GI57R, RI59C, RI9IQ, L198W, A232E, T262A, N387H, and A439S on CFTRAF508 and Tyrosinase C89R of ERAD substrates. The studies indicated that CFTR $\Delta$ F508 and Tyrosinase C89R substrates accumulated in mutant p97 / VCP-expressing cells compared to wild-type p97/VCP. ${ }^{44}$ This result suggested that the deterioration of the ERAD pathway was the result of $\mathrm{p} 97$ / VCP mutations and was a common feature of the molecular pathogenesis of IBMPFD. In the ectopic expression trial, the interaction patterns and subcellular localization of the PI37L mutant have been significantly altered. In the studies carried out with U2OS and $\mathrm{C} 2 \mathrm{Cl} 2$ cells, it was seen that PI37L mutant showed a profile of abundant small, discrete, and punctate cytoplasmic structures in cells unlike R95C and RI55C mutants. It was also determined that PI37L mutant passed to the insoluble protein phase compared to other p97 / VCP mutant proteins. The ability of PI37L mutant to bind with ubiquitin was found to have largely lost. However, the interaction of R95G and RI55C mutants with ubiquitin was found to have enhanced. ${ }^{44} \mathrm{gp} 78$ is an E3 ubiquitin ligase enzyme located in the ER lumen, which is responsible for the ubiquitination of substrate molecules associated with ERAD. The studies have shown that p97 NCP interacts with the VIM motif of gp78. ${ }^{45}$ Similar to the interactions of R95G and RI55C mutants with ubiquitin, their interaction with gp78 and cofactor proteins Ufdl-Npl4 have been reported to have enhanced. Moreover, the interaction of PI37L mutant with gp78 was found to be similar to wild type p97/
VCP. It has also been reported that the ability of the PI37L mutant to interact with Ufdl was lost. Similar results were observed in p47 interaction, a well-known cofactor of p97/ VCP. PI37L was found to have lost the ability to interact with $\mathrm{p} 47 .{ }^{44}$ Johnson et al. showed in vivo studies with RI55H, RI9IQ, and $\mathrm{A} 232 \mathrm{E}$ that these mutant proteins disrupt the formation of lysosomal tubules. Impaired lysosomal tubular formation is associated with deformed autophagosome-lysosome fusion, increased cytoplasmic ubiquitin aggregates, damaged mitochondria, and impaired muscle function. ${ }^{46}$ In another study, PI37L was reported to be the target of the autophagic process. PI37L was selectively degraded by autophagy due to lysosomal activity under basal cellular conditions. In a study carried out with U2OS and PC-12 cells, PI37L was found to stimulate autophagosome and autolysosome formations. At the same time, this study determined that protein aggregates were created by GI57R mutant-induced autophagy and were eliminated by autophagic degradation. These results suggest that some p97 / VCP mutants are a target of the autophagic destruction pathway. ${ }^{47}$

Although the effects of some p97 / VCP mutations have been identified in the pathophysiology of IBMPFD at molecular levels, the vast majority of these p97/ VCP mutations remain unknown.

\section{METHODS}

A literature review was conducted concerning studies about IBMPFD, p97 NCP, and its mutations related to IBMPFD pathogenesis, and also underlying details of molecular pathogenesis mechanism. The current IBMPFD related mutations and their association with $\mathrm{p} 97 / \mathrm{VCP}$ were searched by using National Center for Biotechnology Information (NCBI) and Online Mendelian Inheritance in Man (OMIM) databases.

\section{CONCLUSION}

IBMPFD is a multiple system disease, which three different pathologies are observed simultaneously. The underlying cause of IBMPFD is the mutations observed in the gene called VCP. In the light of the above-mentioned literature, it is clear that 997 / VCP mutations associated with IBMPFD promote the pathogenesis process through a variety of molecular properties in cells. Characterizing all interaction patterns of p97 / VCP by means of mechanistically approaches is important in order to understand the mechanisms underlying the p97/VCP mutation, which is associated with IBMPFD pathogenesis in patients, and develop new generation treatment approaches.

\section{Informed Consent: N/A}

Peer-review: Externally peer-reviewed.

Author Contributions: Concept - Y.E.; Design - Y.E.; Supervision - Y.E.; Data collection and processing - Y.E.; Analysis and interpretation - Y.E.; Literature review - Y.E.; Writer - Y.E.; Critical review - Y.E.

Conflict of Interest: The authors have no conflicts of interest to declare.

Financial Disclosure: The authors declared that this study has received no financial support.

\section{REFERENCES}

I. Mengel D, Librizzi D, Schoser B, et al. Inclusion body myopathy, Paget's disease, and fronto-temporal dementia: A VCP-related 
multi-systemic proteinopathy. Fortschr Neurol Psychiatr. 2018;86(7): 434-438.

2. Palmio J, Sandell S, Suominen T, et al. Distinct distal myopathy phenotype caused by VCP gene mutation in a Finnish family. Neuromuscul Disord. 2011;21(8):55I-555. [CrossRef]

3. Ju JS, Weihl CC. Inclusion body myopathy, Paget's disease of the bone and fronto-temporal dementia: A disorder of autophagy. Hum Mol Genet. 2010;19(I):38-45.

4. Kimonis V. 2019. Inclusion Body Myopathy with Paget Disease of Bone and/or Frontotemporal Dementia. Seattle, WA: GeneReviews ${ }^{8}$.

5. Mérai Z, Chumak N, García-Aguilar M, et al. The AAA-ATPase molecular chaperone $\mathrm{Cdc} 48 / \mathrm{p} 97$ disassembles sumoylated centromeres, decondenses heterochromatin, and activates ribosomal RNA genes. Proc Natl Acad Sci USA. 2014;III(45):16166-16171. [CrossRef]

6. Panginikkod S, Musa R. Inclusion Body Myositis. Treasure Island, FL, StatPearls, 2019.

7. Rodriguez-Ortiz CJ, Hoshino H, Cheng D, et al. Neuronal-specific overexpression of a mutant valosin-containing protein associated with IBMPFD promotes aberrant ubiquitin and TDP-43 accumulation and cognitive dysfunction in transgenic mice. Am J Pathol. 2013;183(2):504-515. [CrossRef]

8. Yi L, Donsante $A$, Kennerson $M L$, et al. Altered intracellular localization and valosin-containing protein (p97 VCP) interaction underlie ATP7A-related distal motor neuropathy. Hum Mol Genet. 2012;2I(8):1794-I807. [CrossRef]

9. Hübbers CU, Clemen CS, Kesper K, et al. Pathological consequences of VCP mutations on human striated muscle. Brain. 2007;130(2):381-393. [CrossRef]

10. Al-Obeidi E, Al-Tahan S, Surampalli A, et al. Genotype-phenotype study in patients with valosin-containing protein mutations associated with multisystem proteinopathy. Clin Genet. 2018;93(I):II9-125. [CrossRef]

II. Fernández-Sáiz $V$, Buchberger A. Imbalances in p97 co-factor interactions in human proteinopathy. EMBO Rep. 20I0;II(6):479485. [CrossRef]

12. Guo X, Zhao Z, Shen H, et al. VCP myopathy: A family with unusual clinical manifestations. Muscle Nerve. 2019;59(3):365-369. [CrossRef]

13. Janiesch PC, Kim J, Mouysset J, et al. The ubiquitin-selective chaperone CDC-48/p97 links myosin assembly to human myopathy. Nat Cell Biol. 2007;9(4):379-390. [CrossRef]

14. Ju JS, Miller SE, Hanson Pl, et al. Impaired protein aggregate handling and clearance underlie the pathogenesis of p97 / VCP-associated disease. J Biol Chem. 2008;283(44):30289-30299. [CrossRef]

15. Erzurumlu Y, Ilhan R, Gozen O, et al. VCP (valosin containing protein). Atlas Genet Cytogenet Oncol Haematol. 2013;17(8):550-556.

16. Hirabayashi M, Inove K, Tanaka K, et al. VCP/p97 in abnormal protein aggregates, cytoplasmic vacuoles, and cell death, phenotypes relevant to neurodegeneration. Cell Death Differ. 2001;8(I0):977-984. [CrossRef]

17. Woodman PG. p97, a protein coping with multiple identities. J Cell Sci. 2003;Il6:4283-4290. [CrossRef]

18. Al-Tahan S, Al-Obeidi E, Yoshioka $H$, et al. Novel valosincontaining protein mutations associated with multisystem proteinopathy. Neuromuscul Disord. 2018;28(6):49I-50I. [CrossRef]

19. Bodnar NO, Kim KH, Ji Z, et al. Structure of the Cdc48 ATPase with its ubiquitin-binding cofactor Ufdl-Npl4. Nat Struct Mol Biol. 2018;25(7):616-622. [CrossRef]

20. Huang R, Ripstein ZA, Rubinstein JL, et al. Cooperative subunit dynamics modulate p97 function. Proc Natl Acad Sci USA. 2019;116(I):158-167. [CrossRef]
21. Haines DS. p97-containing complexes in proliferation control and cancer: Emerging culprits or guilt by association? Genes Cancer. 2010;I(7):753-763. [CrossRef]

22. Lucente $G$, Almendrote $M$, Ramos-Fransi $A$, et al. A novel mutation in the valosin-containing-protein gene found in a Spanish family. $J$ Neurol Sci. 2018;391:||2-II3. [CrossRef]

23. Zhang $X$, Gui $L$, Zhang $X$, et al. Altered cofactor regulation with disease-associated p97 /VCP mutations. Proc Natl Acad Sci USA. 20I5;II2(I4):EI705-EI7|4. [CrossRef]

24. Bulfer SL, Chou TF, Arkin MR. p97 disease mutations modulate nucleotide-induced conformation to alter protein-protein interactions. ACS Chem Biol. 2016; II(8):2112-2116. [CrossRef]

25. DeLaBarre B. Central pore residues mediate the p97/VCP activity required for ERAD. Mol Cell. 2006;22:45I-462. [CrossRef]

26. Nishimura N, Radwan MO, Amano M, et al. Novel p97/VCP inhibitor induces endoplasmic reticulum stress and apoptosis in both bortezomib-sensitive and -resistant multiple myeloma cells. Cancer Sci. 2019;1I0(10):3275-3287. [CrossRef]

27. Liu $Y$, Ye Y. Roles of p97-associated deubiquitinases in protein quality control at the endoplasmic reticulum. Curr Protein Pept Sci. 2012;13(5):436-446. [CrossRef]

28. Araki K, Nagata K. Protein folding and quality control in the ER. Cold Spring Harb Perspect Biol. 20II;3(II):a007526-a007526.

29. Weihl CC, Dalal S, Pestronk A, et al. Inclusion body myopathyassociated mutations in p97 / VCP impair endoplasmic reticulumassociated degradation. Hum Mol Genet. 2006;15(2):189-199. [CrossRef]

30. Rohrer JD, Warren JD, Reiman $D$, et al. A novel exon 2 I27V VCP variant is associated with dissimilar clinical syndromes. J Neurol. 20ll;258(8):1494-1496. [CrossRef]

31. Majounie E, Traynor BJ, Chio? A, et al. Mutational analysis of the VCP gene in Parkinson's disease. Neurobiol Aging. 2012;33(209):e20l-209.202.

32. Inove M, Lida A, Hayashi $S$, et al. Two novel VCP missense variants identified in Japanese patients with multisystem proteinopathy. Hum Genome Var. 2018;5:9. [CrossRef]

33. Guyant-Maréchal L, Laquerriere A, Duyckae?rts C, et al. Valosincontaining protein gene mutations: Clinical and neuropathologic features. Neurology. 2006;67:644-65I. [CrossRef]

34. Watts GD, Wymer J, Kovach MJ, et al. Inclusion body myopathy associated with Paget disease of bone and frontotemporal dementia is caused by mutant valosin-containing protein. Nat Genet. 2004;36:377-38I. [Database] [CrossRef]

35. Kimonis VE, Mehta SG, Fulchiero EC, et al. Clinical studies in familial VCP myopathy associated with Paget disease of bone and frontotemporal dementia. Am J Med Genet. 2008b;146A:745-757. [CrossRef]

36. Kimonis $V E$, Fulchiero $E$, Vesa J, et al. $V C P$ disease associated with myopathy, Paget disease of bone and frontotemporal dementia: Review of a unique disorder. Biochim Biophys Acta. 2008a;1782:744-748. [CrossRef]

37. Gu JM, Ke YH, Yue $H$, et al. A novel VCP mutation as the cause of atypical IBMPFD in a Chinese family. Bone. 2013;52:9-16. [CrossRef]

38. Jerath NU, Crockett CD, Moore SA, et al. Rare manifestation of a c.290 C> T, p.Gly97Glu VCP mutation. Case Rep Genet. 2015;2015:I5.

39. Matsubara S, Shimizu T, Komori T, et al. Nuclear inclusions mimicking poly(a)-binding protein nuclear I inclusions in a case of inclusion body myopathy associated with Paget disease of bone and frontotemporal dementia with a novel mutation in the valosin-containing protein gene. Neuromuscul Disord. 2016;26:436-440. [CrossRef]

40. Stojkovic $\mathrm{T}$, Hammouda el $\mathrm{H}$, Richard $\mathrm{P}$, et al. Clinical outcome in 19 French and Spanish patients with Valosin-containing protein 
myopathy associated with Paget's disease of bone and frontotemporal dementia. Neuromuscul Disord. 2009;19:316-323. [CrossRef]

41. Kwan Tang W, Xia D. Mutations in the human AAA+ chaperone p97 and related diseases. Front Mol Biosci. 2016;3:79.

42. Watts GD, Thomasova D, Ramdeen SK, et al. Novel VCP mutations in inclusion body myopathy associated with Paget disease of bone and frontotemporal dementia. Clin Genet. 2007;72(5):420426. [CrossRef]

43. Issacson RL, Pye VE, Simpson $P$, et al. Detailed structural insights into the p97-Npl4-Ufdl interface. J Biol Chem. 2007;282(29):2136l21369. [CrossRef]
44. Erzurumlu Y, Kose FA, Gozen O, et al. A unique IBMPFD-related P97/VCP mutation with differential binding pattern and subcellular localization. Int J Biochem Cell Biol. 2013;45(4):773-782. [CrossRef]

45. Wang $Y$, Ballar $P$, Zhong $Y$, et al. SVIP induces localization of $\mathrm{p} 97 /$ $\checkmark C P$ to the plasma and lysosomal membranes and regulates autophagy. PLoS One. 2011;6(8):e24478. [CrossRef]

46. Johnson $A E$, Shu $H$, Hauswirth $A G$, et al. VCP-dependent muscle degeneration is linked to defects in a dynamic tubular lysosomal network in vivo. Elife. 2015;4:07366.

47. Bayraktar $\mathrm{O}$, Oral $\mathrm{O}$, Kocaturk NM, et al. IBMPFD disease-causing mutant VCP/p97 proteins are targets of autophagic-lysosomal degradation. PLoS One. 2016;II(I0):e0164864. [CrossRef] 